\title{
Longitudinal neuroimaging and neuropsychological profiles of frontotemporal dementia with C9ORF72 expansions
}

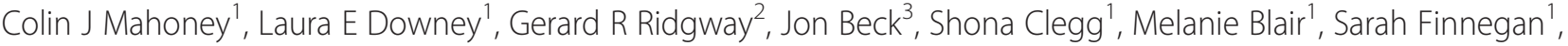 \\ Kelvin K Leung ${ }^{1}$, Tom Yeatman ${ }^{1}$, Hannah Golden ${ }^{1}$, Simon Mead ${ }^{3}$, Jonathan D Rohrer ${ }^{1}$, Nick C Fox ${ }^{1}$ and \\ Jason D Warren ${ }^{1 *}$
}

\begin{abstract}
Introduction: Frontotemporal dementia (FTD) is a common cause of early-onset dementia with a significant genetic component, as underlined by the recent identification of repeat expansions in the gene C9ORF72 as a major cause of FTD and motor neuron disease. Understanding the neurobiology and clinical phenomenology of this novel mutation is currently a major research focus. However, few data are available concerning the longitudinal evolution of this genetic disease. Here we present longitudinal neuropsychological and neuroimaging data on a cohort of patients with pathological repeat expansions in C9ORF72.
\end{abstract}

Methods: Following a review of the University College London FTD DNA database, 20 cases were retrospectively identified with a C9ORF72 expansion. Twelve cases had longitudinal neuropsychology data available and six of these cases also had longitudinal volumetric brain magnetic resonance imaging. Cortical and subcortical volumes were extracted using FreeSurfer. Rates of whole brain, hemispheric, cerebellar and ventricular change were calculated for each subject. Nonlinear fluid registration of follow-up to baseline scan was performed to visualise longitudinal intra-subject patterns of brain atrophy and ventricular expansion.

Results: Patients had low average verbal and performance IQ at baseline that became impaired $(<5$ th percentile) at follow-up. In particular, visual memory, naming and dominant parietal skills all showed deterioration. Mean rates of whole brain atrophy (1.4\%/year) and ventricular expansion (3.2 $\mathrm{ml} /$ year) were substantially greater in patients with the C9ORF72 mutation than in healthy controls; atrophy was symmetrical between the cerebral hemispheres within the C9ORF72 mutation group. The thalamus and cerebellum showed significant atrophy whereas no cortical areas were preferentially affected. Longitudinal fluid imaging in individual patients demonstrated heterogeneous patterns of progressive volume loss; however, ventricular expansion and cerebellar volume loss were consistent findings.

Conclusion: Disease evolution in C9ORF72-associated FTD is linked neuropsychologically with increasing involvement of parietal and amnestic functions, and neuroanatomically with rather diffuse and variable cortical and central atrophy but more consistent involvement of the cerebellum and thalamus. These longitudinal profiles are consistent with disease spread within a distributed subcortical network and demonstrate the feasibility of longitudinal biomarkers for tracking the evolution of the C9ORF72 mutation phenotype.

\footnotetext{
* Correspondence: jason.warren@ucl.ac.uk

'Dementia Research Centre, University College London Institute of

Neurology, London WC1N 3BG, UK

Full list of author information is available at the end of the article
} 


\section{Introduction}

Frontotemporal dementia (FTD) is characterised by early behavioural change and progressive erosion of social cognition associated with frontotemporal lobar degeneration [1]. A substantial number of cases of FTD have a familial basis [2], and an expanded hexanucleotide (GGGGCC) repeat insertion in a noncoding promoter region of ORF 72 of chromosome 9 (C9ORF72) was recently identified as an important cause of FTD and motor neuron disease $[3,4]$. Recent reports of C9ORF72 mutations suggest these are a common cause of FTD and motor neuron disease, representing approximately one-third of all cases due to genetic mutations $[5,6]$, of comparable frequency to mutations in progranulin $(G R N)$ and micro-tubule protein tau $(M A P T)$ as a cause of autosomal dominant FTD [6].

Clinically, C9ORF72 expansions have been associated with a behavioural dysexecutive phenotype but also with notable early features including psychosis and anxiety as well as impaired episodic memory [6,7]. Individual crosssectional magnetic resonance imaging (MRI) has revealed a highly variable imaging phenotype with involvement of frontal, temporal and parietal cortices, and limited previous longitudinal data have suggested similar rates of whole brain atrophy in C9ORF72 and MAPT mutation cases [6]. Group-level cross-sectional imaging studies have confirmed this distributed pattern of atrophy as well as emphasising additional cerebellar and subcortical involvement $[6,8,9]$. Both imaging and clinical studies suggest that the neurodegenerative process associated with the C9ORF72 expansion is rather diffuse [10]. Whilst early symptoms are most in keeping with frontal lobe dysfunction, parietal dysfunction becomes more apparent as the disease progresses [6]. These clinical features suggest that the disease may propagate along a rostrocaudal gradient, perhaps spreading via a distributed brain network. Understanding the clinical and radiological evolution of C9ORF72-associated FTD is an important issue. Detailed studies of longitudinal imaging profiles and neuropsychological changes associated with C9ORF72 mutations remain limited: longitudinal studies may enable evaluation of candidate biomarkers both for diagnosis and future clinical trials of disease-modifying agents. More fundamentally, the concept of network-led degeneration is gaining currency as an important general theme in neurodegeneration [11] and C9ORF72-associated FTD, as a novel genetic proteinopathy, may offer fresh insights into the mechanisms of neurodegenerative disease propagation.

Here we present longitudinal data on a cohort of patients with FTD associated with C9ORF72 expansions. We detail profiles of neuropsychological progression, rates of whole brain, cerebellar and subcortical atrophy and anatomical profiles of disease progression using nonlinear fluid registration of serial MRI.

\section{Methods \\ Case ascertainment}

Twenty cases from a previously published DNA cohort comprising 227 cases within the frontotemporal lobar degeneration spectrum [6] were found to harbour a C9ORF72 expansion using the repeat-primed PCR as previously published [4]. Mutations were called where more than 30 repeats were shown consistently. For the purpose of reporting longitudinal change only cases with a minimum of two neuropsychological assessments or volumetric MRI scans were included. In total 12 individuals (mean age 59.4 years ( \pm 6.8 years), seven male) had longitudinal neuropsychological data and six of these cases (mean age 62.7 years ( \pm 7 years), five male) also had longitudinal volumetric MRI scans. All cases identified had been assessed in the Specialist Cognitive Disorders Clinic at the National Hospital for Neurology and Neurosurgery by an experienced cognitive neurologist and all met current consensus criteria for a diagnosis of behavioural variant FTD [1]. Two cases had additional clinical features of motor neuron disease at presentation.

The study was approved by the local research ethics committee under Declaration of Helsinki guidelines and all subjects gave informed consent for participation.

\section{Neuropsychological assessment}

The mean duration between serial neuropsychological assessments was 1.4 years ( \pm 0.7 years). General intellectual function was assessed using the Wechsler Adult Intelligence Scale - Revised or the Wechsler Abbreviated Scale of Intelligence [11,12]. Executive function was assessed using the Weigl test, the Stroop colour-word test or the Hayling test [13-15]. Verbal memory and visual memory were assessed with the Recognition Memory Test for words and faces respectively [16]. Naming was assessed using the Graded Naming Test or the Oldfield Naming Test $[17,18]$. Visuospatial and visual perception skills were assessed using subsets of the Visual Object and Spatial Perception battery [19]. Calculation and spelling were assessed with the Graded Difficulty Arithmetic test [20] and the Baxter Spelling tests [21] respectively. Raw scores were converted into percentiles for reporting.

\section{Brain image acquisition and processing}

Serial $\mathrm{T}_{1}$-weighted magnetic resonance volumetric brain MRI was performed using a Magnetization Prepared Rapid Gradient Echo sequence: three studies were acquired on a $1.5 \mathrm{~T}$ GE Signa scanner (General Electric Milwaukee, WI, USA) $(256 \times 256$ matrix; $1.5 \mathrm{~mm}$ slice thickness) and three studies acquired on a $3.0 \mathrm{~T}$ Siemens 
Trio scanner (Siemens, Germany) $(256 \times 256$ matrix; 1.1 $\mathrm{mm}$ slice thickness). Patient data were compared with data from 15 age-matched (mean age 57.7 years; 10 male, five female) healthy controls with two volumetric MRI scans (12 controls on the $1.5 \mathrm{~T}$ scanner, three controls on the 3.0T scanner). The mean duration between scans was 1.0 years $( \pm 0.2$ years $)$ for patients and 1.6 years ( \pm 0.8 years) for controls. All images were visually inspected for alternative pathologies and motion artefact. Whole brain, ventricular and cerebellar segmentation was performed by an experienced segmentor using a semi-automated technique using the MIDAS software package [22]. Scans underwent affine registration to spatially align the repeat scan to baseline. Rates of whole brain and cerebellar atrophy and ventricular expansion were calculated using the boundary shift integral (BSI), utilising the more robust KN-BSI methodology to provide automatic quantification of volume change[23]. Rates of change are expressed as the percentage loss from baseline volume and adjusted to an annualised rate according to the interval. Scans were registered into standard space for ventricular and hemispheric segmentation. Ventricular regions included the lateral ventricles and temporal horn of the lateral ventricles but excluded the third and fourth ventricles. Visualised cerebellar regions were dissected from the brainstem at mid-pontine level and images underwent further manual editing in coronal and sagittal planes to remove any remaining areas of brainstem. Right and left cerebral hemispheric volumes were calculated by dividing the brain along the mid-sagittal section. Finally, total intracranial volumes were calculated by summing grey matter, white matter and cerebrospinal fluid volumes acquired using the New Segment toolbox within Statistical Parametric Mapping $8[24,25]$.

Cortical and subcortical regional volumes were obtained from each subject's baseline and repeat volumetric MRI images using FreeSurfer (v5.1) running the automated longitudinal processing stream [26]. Default parameters were used with the exception of applying custom brain masks defined from the whole brain segmentation step to improve anatomical accuracy. Segmentations were visually inspected and edited where necessary. Volumes from 34 cortical regions following the Desikan atlas [27] and six subcortical regions (thalamus, caudate, putamen, globus pallidus, amygdala, hippocampus) were extracted for each subject and time point.

Following affine registration and bias correction of scan pairs, each scan set underwent cropping using subjectspecific masks to exclude nonbrain regions. Fluid registration was performed to visualise intra-subject changes in brain morphology [28]. Briefly this involves nonlinear warping of each individual's repeat scan to match their baseline scan, generating a deformation field for each subject that allows visualisation of voxel-level expansion or contraction.

\section{Results}

\section{Neuropsychological findings}

Group-level performance is displayed in Figure 1 and individual data in Table 1. At baseline the mean general intellectual function, as reflected in verbal IQ and performance IQ, was in the low average range (mean baseline verbal IQ $=83( \pm 14)$; mean baseline performance $\mathrm{IQ}=83( \pm 15))$; over the period of follow-up, both verbal IQ and performance IQ became impaired, declining by an average of 11 points (mean follow-up verbal IQ = 72 ( \pm 19$)$; mean follow-up performance IQ = $71( \pm 23)$ ).

Executive function was severely impaired in the majority of subjects at baseline $(7 / 12$ subjects scored $<5$ th percentile on at least one executive measure) and deficits became more frequent over the period of follow-up $(10 / 12<5$ th percentile). Recognition memory was frequently weak at baseline, with deficits in verbal $(7 / 12<5$ th percentile) and visual memory $(8 / 12<5$ th percentile); over the period of follow-up, visual memory deficits became more frequent $(10 / 12<5$ th percentile). Naming was impaired in one-half of the patients at baseline $(6 / 12<5$ th percentile); at follow-up, naming deficits were evident in the majority (8/ $12<5$ th percentile)

Dominant parietal skills were assessed in only five patients longitudinally; however, three had evidence of dyscalculia and/or dysgraphia at baseline and four exhibited at least one of these deficits at follow-up. Visual perceptual functions remained largely stable over the period of follow-up, only one subject becoming impaired.

\section{Neuroimaging findings: atrophy rates}

Individual and group volumetric data and rates of whole brain, hemispheric and ventricular change measured using BSI are displayed in Figure 2 and Table 2. Rates of whole brain atrophy varied widely between subjects; these data have been reported previously for five of the cases in the present series [6]. The most consistent finding (present in 5/6 cases) was an increased rate of ventricular enlargement in patients with a C9ORF72 mutation: patients had a mean annualised ventricular expansion rate of $3.2( \pm 2.0) \mathrm{ml} /$ year compared with controls at 0.7 $\mathrm{ml} /$ year $( \pm 0.6)(P=0.001)$, despite substantial individual variation. Longitudinal cerebellar atrophy was present in the majority of individual patients ( $4 / 6$ cases); the mean annualised rate of cerebellar atrophy in the patients was also significantly higher (1.0\%/year) than in controls $(0.1 \% /$ year; $P=0.02)$. In addition, the mean annualised rate of whole brain atrophy in the patients as measured 


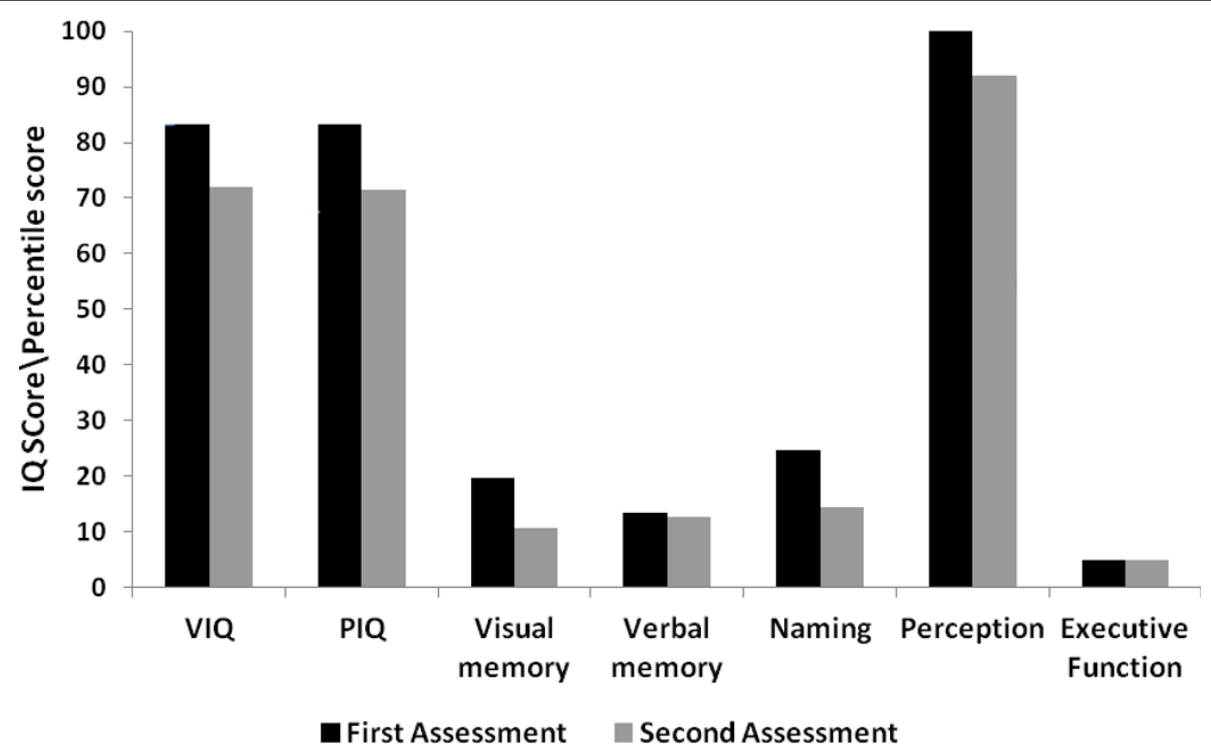

Figure 1 Group-level longitudinal performance on neuropsychological measures. Verbal IQ (VIQ) and performance IQ (PIQ) reported as raw scores. For the purpose of standardising across tests and to visualise changes in performance, raw scores were converted to percentiles. Where a score of 50th to 75th percentile was reported this was converted to the median value (that is, 62.5) for visualisation.

Table 1 Individual neuropsychological data for baseline and follow-up assessments

\begin{tabular}{|c|c|c|c|c|c|c|c|c|c|c|}
\hline Case & Syndrome & $\begin{array}{l}\text { Disease duration } \\
\text { (years) }\end{array}$ & $\begin{array}{l}\text { Assessment } \\
\text { number }\end{array}$ & VIQ & PIQ & RMT-F & RMT-W & Naming & $\begin{array}{l}\text { VOSP } \\
\%\end{array}$ & $\begin{array}{l}\text { Executive } \\
\text { function }^{a}\end{array}$ \\
\hline \multirow[t]{2}{*}{1} & bvFTD & 34.5 & 1 & 106 & 113 & $<5$ th & 5 th to 10 th & 95th & Pass & $<5$ th \\
\hline & & 4.5 & 2 & 110 & 110 & $\begin{array}{l}\text { 2nd to } \\
\text { 10th }\end{array}$ & N/A & $>25$ th & Pass & $<5$ th \\
\hline \multirow[t]{2}{*}{2} & bvFTD & 3 & 1 & 87 & 78 & $<5$ th & $<5$ th & $<5$ th & Pass & $<5$ th \\
\hline & & 4.7 & 2 & 81 & 76 & 25th & $<10$ th & $<1 s t$ & Pass & $<5$ th \\
\hline \multirow[t]{2}{*}{3} & bvFTD & 7 & 1 & 85 & 69 & $\begin{array}{l}\text { 50th to } \\
75 \text { th }\end{array}$ & $\begin{array}{l}\text { 10th to } \\
25 \text { th }\end{array}$ & $<5$ th & Pass & $<5$ th \\
\hline & & 7.7 & 2 & 68 & 71 & $<5$ th & 5 th to 10 th & $\begin{array}{l}\text { 5th to } \\
10 \text { th }\end{array}$ & Fail & $<5$ th \\
\hline \multirow[t]{2}{*}{4} & bvFTD & 3 & 1 & 73 & 78 & $<5$ th & $<5$ th & $\begin{array}{l}\text { 5th to } \\
10 \text { th }\end{array}$ & Pass & $<5$ th \\
\hline & & 4.5 & 2 & NT & NT & $<5$ th & $<5$ th & $<1 s t$ & Pass & Reduced \\
\hline \multirow[t]{2}{*}{5} & bvFTD & 11 & 1 & 116 & 113 & $\begin{array}{l}\text { 50th to } \\
75 \text { th }\end{array}$ & $\begin{array}{l}\text { 75th to } \\
95 \text { th }\end{array}$ & 75th & Pass & Pass \\
\hline & & 14.4 & 2 & 101 & 114 & $\begin{array}{l}50 \text { th to } \\
75 \text { th }\end{array}$ & $>95$ th & $>75$ th & Pass & Pass \\
\hline \multirow[t]{2}{*}{6} & bvFTD & 2.75 & 1 & 69 & 69 & $<5$ th & $<5$ th & $<5$ th & Pass & $<5$ th \\
\hline & & 3.75 & 2 & 57 & 67 & $<5$ th & $<5$ th & $\begin{array}{l}\text { 5th to } \\
\text { 10th }\end{array}$ & Pass & $<5$ th \\
\hline \multirow[t]{2}{*}{7} & bvFTD & 1 & 1 & 80 & 89 & $<5$ th & $<5$ th & 75th & Pass & Borderline \\
\hline & & 2.8 & 2 & 59 & 63 & $<5$ th & $<5$ th & $<5$ th & Pass & $<5$ th \\
\hline \multirow[t]{2}{*}{8} & FTD-MND & 3 & 1 & 69 & 80 & $<5$ th & 5 th to 10 th & $\begin{array}{l}\text { 5th to } \\
\text { 10th }\end{array}$ & Pass & $<5$ th \\
\hline & & 4.2 & 2 & 67 & 65 & $<5$ th & $<5$ th & $\begin{array}{l}\text { 5th to } \\
10 \text { th }\end{array}$ & Pass & $<5$ th \\
\hline \multirow[t]{2}{*}{9} & bvFTD & 2 & 1 & 78 & 76 & $<5$ th & $<5$ th & $>5$ th & Pass & Borderline \\
\hline & & 3 & 2 & 78 & NT & $<5$ th & $<5$ th & $>25$ th & Pass & $<5$ th \\
\hline \multirow[t]{2}{*}{10} & bvFTD & 5 & 1 & 82 & 77 & Normal & $<5$ th & $<5$ th & Pass & Pass \\
\hline & & 6.6 & 2 & 55 & 77 & $<5$ th & $<5$ th & $<1 s t$ & Pass & $<5$ th \\
\hline 11 & FTD-MND & 1.5 & 1 & 84 & 82 & 10th & 5 th to 10 th & $<5$ th & Pass & $<5$ th \\
\hline
\end{tabular}


Table 1 Individual neuropsychological data for baseline and follow-up assessments (Continued)

\begin{tabular}{|c|c|c|c|c|c|c|c|c|c|c|}
\hline & & 2.5 & 2 & 77 & 60 & $<5$ th & $<5$ th & $\begin{array}{l}\text { 5th to } \\
\text { 10th }\end{array}$ & Pass & $<5$ th \\
\hline \multirow[t]{2}{*}{12} & bvFTD & 3 & 1 & 71 & 75 & $<5$ th & $<5$ th & $<5$ th & Pass & Pass \\
\hline & & 3.7 & 2 & 71 & 74 & $<5$ th & $<5$ th & N/A & Pass & Pass \\
\hline
\end{tabular}

Raw scores are displayed for verbal IQ (VIQ) and performance IQ (PIQ); otherwise results are displayed as percentiles. Pass, > 5th percentile; borderline, 5th to 10th percentile. bvFTD, behavioural variant frontotemporal dementia; FTD-MND, frontotemporal dementia-motor neuron disease; N/A, not available; NT, not testable; RMT-F, Recognition Memory Test for faces; RMT-W, Recognition Memory Test for words; VOSP, visual object and space perception battery. ${ }^{\text {aTests }}$ of executive function (Weigl test, Stroop colour-word test or Hayling test).

using KN-BSI (1.4\%) was significantly higher than in controls $(0.4 \% ; P=0.04)$ Mean atrophy rates for the cerebral hemispheres considered separately were similar for each hemisphere (left 2.4\%/year; right 2.1\%/year) and similar to the whole brain atrophy rate; atrophy in individual patients was symmetrical between the hemispheres across the C9ORF72 mutation group (inter-hemispheric volume ratio 0.99 ) and did not become more asymmetric over the follow-up interval.
Neuroimaging findings: cortical and subcortical regions Detailed data on subcortical volume change are displayed in Table 3. Compared with healthy controls, significant subcortical volume loss over time was detected in the C9ORF72 mutation group in the right thalamus $(P=0.006)$, left thalamus $(P=0.03)$ and left globus pallidus $(P=0.04)$. No significant change over time was detected in cortical regions when compared with controls.

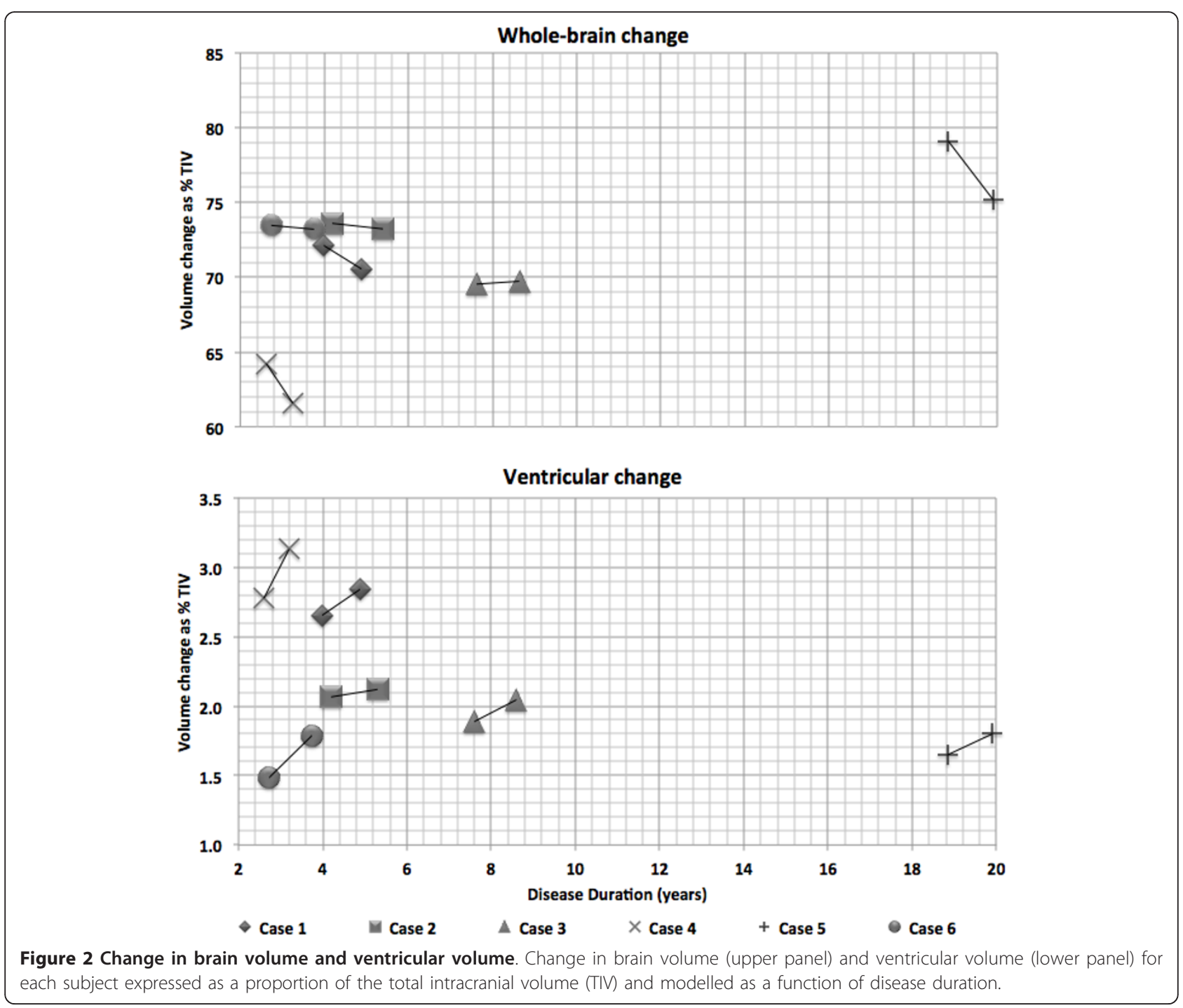


Table 2 Individual patient and group brain magnetic resonance imaging volumetric data

\begin{tabular}{|c|c|c|c|c|c|c|c|c|}
\hline & Case 1 & Case 2 & Case 3 & Case 4 & Case 5 & Case 6 & C9ORF72 & Controls \\
\hline Age at first scan & 63.0 & 59.2 & 65.6 & 70.6 & 66.8 & 50.7 & $62.7(7.0)$ & $58.7(5.8)$ \\
\hline Gender (male:female) & & & & & & & $5: 1$ & $10: 5$ \\
\hline Disease duration (years) & 2.9 & 4.2 & 7.6 & 2.6 & 18.8 & 2.7 & $6.5(6.3)$ & N/A \\
\hline Interscan interval (years) & 0.9 & 1.2 & 1 & 0.6 & 1.1 & 1.1 & $1.0(0.2)$ & $1.6(0.8)$ \\
\hline Total intracranial volume (ml) & 1,624 & 1,835 & 1,854 & 1,368 & 1,686 & 1,465 & $1,639(196)$ & $1,610(144)$ \\
\hline Brain volume, baseline (ml) & 1,171 & 1,351 & 1,290 & 876 & 1,232 & 1,076 & $1,166(171)$ & $1,231(92)$ \\
\hline Brain volume, repeat (ml) & 1,146 & 1,344 & 1,293 & 842 & 1,179 & 1,072 & $1,146(179)$ & $1,226(92)$ \\
\hline Volume change (ml) & 25 & 7 & -3 & 34 & 53 & 4 & $20(22)$ & $5(17)$ \\
\hline Left hemisphere, baseline (ml) & 592 & 675 & 651 & 433 & 623 & 542 & $586(88)$ & $614(56)$ \\
\hline Left hemisphere, repeat (ml) & 579 & 675 & 653 & 415 & 592 & 538 & $575(93)$ & $611(53)$ \\
\hline Right hemisphere, baseline (ml) & 589 & 686 & 646 & 451 & 619 & 542 & $589(84)$ & $612(60)$ \\
\hline Right hemisphere, repeat (ml) & 576 & 681 & 648 & 433 & 595 & 541 & $579(87)$ & $609(54)$ \\
\hline Ventricle volume, baseline (ml) & 44.4 & 38.2 & 35.0 & 40.3 & 27.8 & 21.8 & $34.6(8.4)$ & $21.4(11.4)$ \\
\hline Ventricle volume, repeat (ml) & 47.7 & 39.0 & 37.9 & 44.8 & 29.8 & 26.2 & $37.6(8.3)$ & $22.5(12.2)$ \\
\hline Brain BSI (\%/year) & 1.1 & -0.2 & 0.5 & 4.4 & 1.0 & 1.6 & $1.4(1.6)$ & $0.4(0.3)$ \\
\hline Ventricle expansion (ml/year) & 3.6 & 0.8 & 2.5 & 6.2 & 1.6 & 4.3 & $3.2(2.0)$ & $0.7(0.6)$ \\
\hline Cerebellar BSI (\%/year) & -0.5 & -0.2 & 0.6 & 1.9 & 1.8 & 2.6 & $1.0(1.3)$ & $0.1(0.5)$ \\
\hline Left hemisphere rate (\%/year) & 2.4 & 0.1 & -0.4 & 6.9 & 4.6 & 0.6 & $2.4(2.9)$ & $0.5(1.3)$ \\
\hline Right hemisphere rate (\%/year) & 2.4 & 0.6 & -0.2 & 6.2 & 3.7 & 0.1 & $2.1(2.5)$ & $0.5(1.0)$ \\
\hline
\end{tabular}

Rates of whole brain, hemispheric and ventricular change. Group data show the mean (standard deviation). BSI, boundary-shift integral; C9ORF72, chromosome 9 open reading frame $72 ; \mathrm{N} / \mathrm{A}$, not applicable.

\section{Nonlinear fluid registrations}

Fluid-based nonrigid registrations in individual patients (Figure 3) revealed heterogeneous patterns of whole brain atrophy across subjects. Over the period of follow-up, most patients showed a diffuse but dorsally directed pattern of cerebral parenchymal loss, with more variable involvement of the temporal lobe regions; ventricular expansion and cerebellar volume loss were consistent features. A pattern of generalised progressive atrophy was apparent in Cases 4 to 6; Case 2 had prominent bifrontal volume loss, particularly implicating orbitofrontal cortices; and Cases 1 and 3 had more posterior atrophy, although expansion of the frontal horns of the lateral ventricles was also prominent in Case 1.

\section{Discussion and Conclusion}

We have described longitudinal neuropsychological and neuroimaging features in a cohort of patients with FTD due to a C9ORF72 expansion. Considering the mutation group as a whole, impaired executive function and also episodic memory were early and prominent neuropsychological features. Over follow-up intervals of some 18

Table 3 Subcortical volumes in C9ORF72 mutation and healthy control groups

\begin{tabular}{|c|c|c|c|c|c|c|c|c|}
\hline & & \multicolumn{3}{|l|}{ C9ORF72 } & \multicolumn{4}{|l|}{ Control } \\
\hline & & $\%$ per year & Atrophy (ml/year) & SD & $\%$ per year & Atrophy (ml/year) & SD & $P$ value \\
\hline \multirow[t]{2}{*}{ Thalamus } & Right & 3.4 & 0.16 & 0.17 & -0.2 & -0.02 & 0.08 & 0.006 \\
\hline & Left & 2.5 & 0.23 & 0.29 & -0.3 & -0.02 & 0.09 & 0.03 \\
\hline \multirow[t]{2}{*}{ Globus pallidus } & Right & 1.7 & 0.05 & 0.10 & 0.0 & 0.00 & 0.03 & $n / s$ \\
\hline & Left & 2.9 & 0.03 & 0.14 & -1.1 & -0.02 & 0.06 & 0.04 \\
\hline \multirow[t]{2}{*}{ Caudate } & Right & -2.6 & -0.09 & 0.15 & 0.3 & 0.01 & 0.06 & $n / s$ \\
\hline & Left & 0.7 & 0.02 & 0.07 & 0.0 & 0.00 & 0.13 & $n / s$ \\
\hline \multirow[t]{2}{*}{ Amygdala } & Right & -2.8 & -0.04 & 0.08 & 0.1 & 0.00 & 0.04 & $\mathrm{n} / \mathrm{s}$ \\
\hline & Left & 2.1 & 0.03 & 0.06 & 0.6 & 0.01 & 0.06 & $n / s$ \\
\hline \multirow[t]{2}{*}{ Hippocampus } & Right & 1.7 & 0.07 & 0.08 & 1.0 & 0.04 & 0.06 & $n / s$ \\
\hline & Left & 1.4 & 0.05 & 0.03 & 0.6 & 0.03 & 0.09 & $n / s$ \\
\hline \multirow[t]{2}{*}{ Putamen } & Right & -0.6 & -0.03 & 0.10 & 0.0 & 0.00 & 0.06 & $\mathrm{n} / \mathrm{s}$ \\
\hline & Left & -1.2 & -0.06 & 0.13 & -0.1 & 0.00 & 0.10 & $\mathrm{n} / \mathrm{s}$ \\
\hline
\end{tabular}

Mean annualised rates of change are expressed as a percentage (\%) and as the millilitre (ml) change from baseline. C9ORF72, chromosome 9 open reading frame 72; $\mathrm{n} / \mathrm{s}$, not significant $\mathrm{p}<0.05 ; \mathrm{SD}$, standard deviation. 


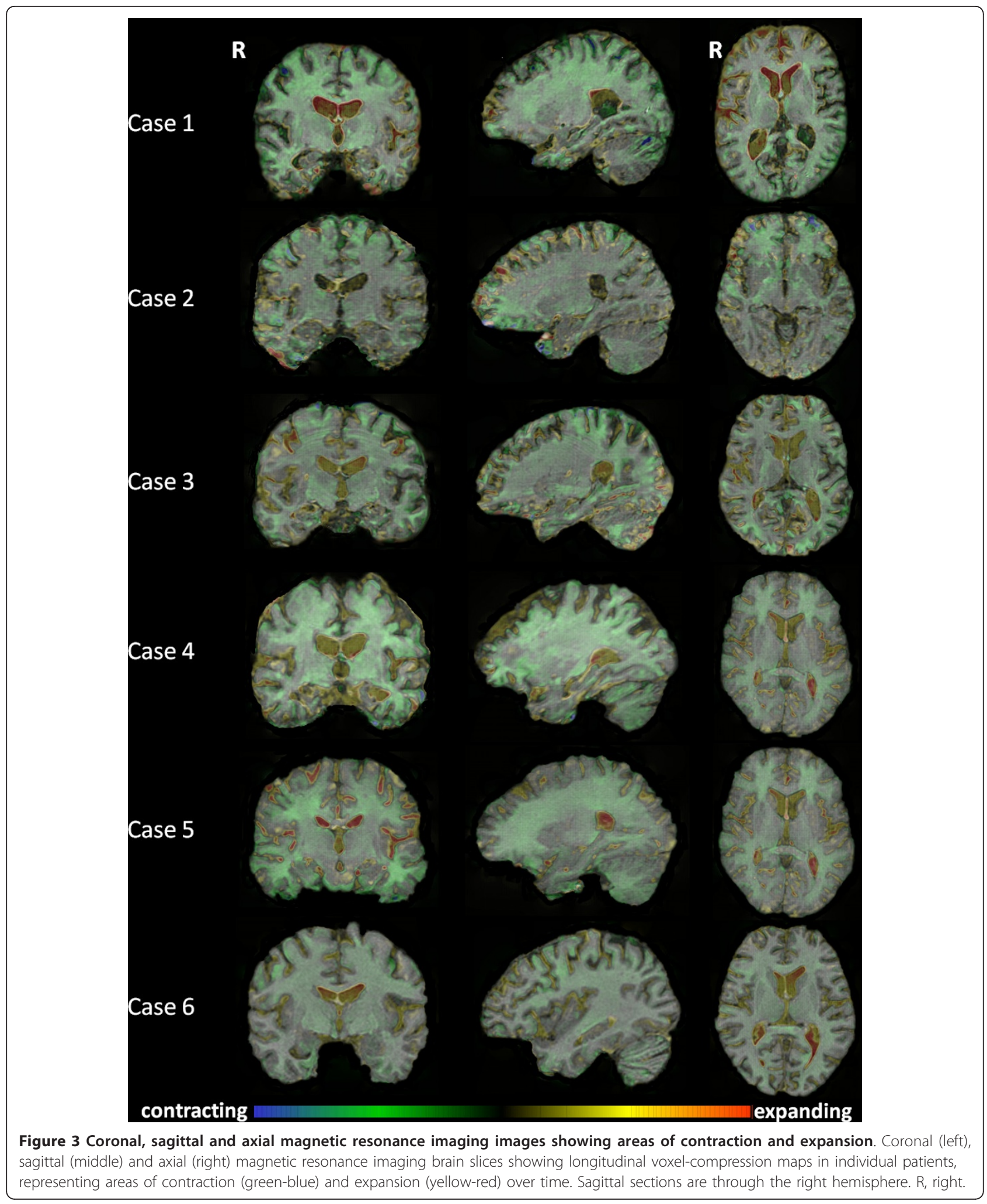

months, there was a significant decline in general intellect and a further decline in visual memory, naming and parietal skills, whereas nondominant parietal (visuoperceptual) functions remained relatively intact. Mean brain atrophy and ventricular expansion rates were increased compared with healthy controls and broadly in line with rates of 
change in previous longitudinal imaging studies of FTD $[29,30]$, although there was substantial diversity across the group. Increased rates of ventricular expansion were consistently observed and may be a candidate biomarker of disease evolution associated with the C9ORF72 mutation. Cerebellar atrophy was also a relatively prominent feature in the C9ORF72 mutation group, with an approximately 10 -fold increase in mean atrophy rate compared with controls. Notably, no specific cortical region appeared disproportionately affected; however, subcortical structures including the thalamus and globus pallidus showed mean rates of atrophy around three times greater than controls. Unlike certain other genetic variants of FTD, notably GRN [31], hemispheric atrophy remained largely symmetrical. In further contrast to previous neuroimaging findings in association with mutations of GRN (asymmetric frontotemporo-parietal atrophy) and MAPT (antero-medial temporal lobe atrophy) [9], individual atrophy profiles in this C9ORF72 mutation cohort were highly variable (Figure 2) - some patients showing chiefly frontal volume loss, whilst others showed relatively more posterior volume loss. Cerebellar atrophy was a relatively consistent feature in individual cases here, although whether this is truly a signature of C9ORF72-associated FTD requires substantiation in larger patient cohorts from different centres.

The evolution of cognitive deficits here suggests a distributed disease process implicating frontal, temporal and parietal cortices, particularly in the dominant hemisphere. Degeneration of a distributed subcortical network might reconcile this neuropsychological profile with the rather variable and diffuse profiles of brain atrophy observed here. Degeneration of thalamus, cerebellum and thalamic and frontal white matter tracts has been identified previously in cross-sectional imaging studies of C9ORF72 expansions $[6,8,9]$. In the present study, we provide further evidence that the pathophysiological mechanisms of C9ORF72-associated FTD target subcortical networks: rates of thalamic and cerebellar atrophy and ventricular expansion were disproportionately increased relative to whole brain atrophy rates, consistent with involvement of subcortical structures and pathways [32]. The involvement of the globus pallidus observed here is in line with the development of extrapyramidal symptoms in a substantial proportion of C9ORF72 cases in other series [33], although our patients did not manifest clear-cut features of Parkinsonism. The thalamus, globus pallidus and cerebellum together act as key hubs coordinating distributed cortico-subcortical circuits and the cognitive operations they mediate [34,35]. Early involvement of such hub regions and projections could facilitate diffusive spread of the molecular pathology responsible for the brain degeneration associated with C9ORF72 expansions [36-38] and might be anticipated to lead to rapid clinical evolution, although the very wide range of clinical disease durations among individual patients with a C9ORF72 mutation remains an important unsolved problem. Both the thalamus and cerebellum have been previously implicated in cross-sectional neuroimaging work in C9ORF72-associated FTD [6]. The increased prevalence of cerebellar p62 inclusions with C9ORF72 expansions compared with other pathologically proven cases of FTD further supports the role of the cerebellum as an important anatomical nidus of C9ORF72associated pathology [6,39].

This study has a number of limitations. Case numbers here were relatively small and individual variation was substantial; larger (ideally, multicentre) longitudinal studies are required to establish the true range of cognitive and neuroimaging features associated with C9ORF72associated FTD and to evaluate candidate biomarkers. The historical nature of the present cohort was a particular limitation on the systematic analysis of behavioural deficits; for example, the nature of the naming impairment here remains ill-defined, and this could in principle reflect primary word retrieval, semantic or mixed deficits. Inclusion of presymptomatic carriers in future studies may allow the earliest behavioural and neuroimaging markers of disease onset to be determined. The specificity of any candidate biomarkers will only be established by comparisons with other genetic and sporadic forms of FTD. We argue that future work should particularly target subcortical (including cerebellar) structures and cognitive functions in the C9ORF72 mutation group, incorporating neuroimaging modalities that capture white matter disintegration: although any synthesis must be preliminary, we interpret the present findings as further circumstantial evidence that a distributed corticosubcortical network is integral to the phenotypic expression of C9ORF72-associated FTD.

\section{Abbreviations}

BSI: boundary shift integral; C9ORF72: chromosome 9 open reading frame 72; FTD: frontotemporal dementia; GRN: progranulin; MAPT: micro-tubule protein tau; MRI: magnetic resonance imaging; ORF: open reading frame; PCR: polymerase chain reaction.

\section{Acknowledgements}

The authors thank all subjects and their families for their participation. This work was undertaken at University College London Hospitals and University College London who received a proportion of funding from the Department of Health's NIHR Biomedical Research Centres funding scheme including a Biomedical Research Unit in dementia. The Dementia Research Centre is an Alzheimer's Research UK Co-ordinating Centre. This work was also funded by the Medical Research Council UK and by the Wellcome Trust. CJM is supported by an MRC programme grant. NCF is a NIHR senior investigator and MRC Senior Clinical Fellow. JDW is supported by a Wellcome Trust Senior Clinical Fellowship (091673/Z/10/Z).

\section{Author details}

${ }^{1}$ Dementia Research Centre, University College London Institute of Neurology, London WC1N 3BG, UK. ${ }^{2}$ Wellcome Trust Centre for Neuroimaging, University College London Institute of Neurology, London 
WC1N 3BG, UK. ${ }^{3}$ MRC Prion Unit, University College London Institute of Neurology, London WC1N 3BG, UK.

\section{Authors' contributions}

CJM contributed to the conception and design of this study, data collection, data analysis and drafting the manuscript. LED contributed to the data collection and data analysis. GRR contributed to data analysis and drafting of the manuscript. JB contributed to the genetic analysis of subjects. SC, MB, SF, KKL and TY contributed to data analysis. HG contributed to data collection. SM contributed to genetic analysis of subjects. JDR contributed to data collection. NCF contributed to conception and design of the study and review of the manuscript. JDW contributed to the conception and design of this study and drafting and critical revision of the manuscript. All authors read and approved the final manuscript.

\section{Competing interests}

The authors declare that they have no competing interests.

Received: 31 May 2012 Revised: 21 August 2012

Accepted: 31 August 2012 Published: 24 September 2012

\section{References}

1. Rascovsky K, Hodges JR, Knopman D, Mendez MF, Kramer JH, Neuhaus J, van Swieten JC, Seelaar H, Dopper EGP, Onyike CU, Hillis AE, Josephs KA, Boeve BF, Kertesz A, Seeley WW, Rankin KP, Johnson JK, Gorno-Tempini ML, Rosen H, Prioleau-Latham CE, Lee A, Kipps CM, Lillo P, Piguet O, Rohrer JD, Rossor MN, Warren JD, Fox NC, Galasko D, Salmon DP, et al: Sensitivity of revised diagnostic criteria for the behavioural variant of frontotemporal dementia. Brain 2011, 134:2456-2477.

2. Rohrer JD, Guerreiro R, Vandrovcova J, Uphill J, Reiman D, Beck J, Isaacs AM, Authier A, Ferrari R, Fox NC, Mackenzie IRA, Warren JD, de Silva R, Holton J, Revesz T, Hardy J, Mead S, Rossor MN: The heritability and genetics of frontotemporal lobar degeneration. Neurology 2009, 73:1451-1456.

3. Dejesus-Hernandez M, Mackenzie IR, Boeve BF, Boxer AL, Baker M, Rutherford NJ, Nicholson AM, Finch NA, Flynn H, Adamson J, Kouri N, Wojtas A, Sengdy P, Hsiung G-YR, Karydas A, Seeley WW, Josephs KA, Coppola G, Geschwind DH, Wszolek ZK, Feldman H, Knopman DS, Petersen RC, Miller BL, Dickson DW, Boylan KB, Graff-Radford NR, Rademakers R: Expanded GGGGCC hexanucleotide repeat in noncoding region of C9ORF72 causes chromosome 9p-linked FTD and ALS. Neuron 2011, 72:245-256.

4. Renton AE, Majounie E, Waite A, Simón-Sánchez J, Rollinson S, Gibbs JR, Schymick JC, Laaksovirta H, van Swieten JC, Myllykangas L, Kalimo H, Paetau A, Abramzon Y, Remes AM, Kaganovich A, Scholz SW, Duckworth J Ding J, Harmer DW, Hernandez DG, Johnson JO, Mok K, Ryten M, Trabzuni D, Guerreiro RJ, Orrell RW, Neal J, Murray A, Pearson J, Jansen IE, et al: A hexanucleotide repeat expansion in C9ORF72 is the cause of chromosome 9p21-linked ALS-FTD. Neuron 2011, 72:257-268.

5. Majounie E, Renton AE, Mok K, Dopper EGP, Waite A, Rollinson S, Chiò A, Restagno G, Nicolaou N, Simon-Sanchez J, van Swieten JC, Abramzon Y, Johnson JO, Sendtner M, Pamphlett R, Orrell RW, Mead S, Sidle KC, Houlden H, Rohrer JD, Morrison KE, Pall H, Talbot K, Ansorge O, Hernandez DG, Arepalli S, Sabatelli M, Mora G, Corbo M, Giannini F, et al: Frequency of the C9orf72 hexanucleotide repeat expansion in patients with amyotrophic lateral sclerosis and frontotemporal dementia: a crosssectional study. Lancet Neurol 2012, 11:323-330.

6. Mahoney CJ, Beck J, Rohrer JD, Lashley T, Mok K, Shakespeare T, Yeatman T, Warrington EK, Schott JM, Fox NC, Rossor MN, Hardy J, Collinge J, Revesz T, Mead S, Warren JD: Frontotemporal dementia with the C9ORF72 hexanucleotide repeat expansion: clinical, neuroanatomical and neuropathological features. Brain 2012, 135:736-750.

7. Snowden JS, Rollinson S, Thompson JC, Harris JM, Stopford CL, Richardson AMT, Jones M, Gerhard A, Davidson YS, Robinson A, Gibbons L, Hu Q, DuPlessis D, Neary D, Mann DMA, Pickering-Brown SM: Distinct clinical and pathological characteristics of frontotemporal dementia associated with C9ORF72 mutations. Brain 2012, 135:693-708.

8. Boxer AL, Mackenzie IR, Boeve BF, Baker M, Seeley WW, Crook R, Feldman H, Hsiung G-YR, Rutherford N, Laluz V, Whitwell J, Foti D, McDade E, Molano J, Karydas A, Wojtas A, Goldman J, Mirsky J, Sengdy P, Dearmond S, Miller BL, Rademakers R: Clinical, neuroimaging and neuropathological features of a new chromosome 9p-linked FTD-ALS family. J Neurol Neurosurg Psychiatry 2011, 82:196-203.

9. Whitwell JL, Weigand SD, Boeve BF, Senjem ML, Gunter JL, DeJesusHernandez M, Rutherford NJ, Baker M, Knopman DS, Wszolek ZK, Parisi JE, Dickson DW, Petersen RC, Rademakers R, Jack CR Jr, Josephs KA: Neuroimaging signatures of frontotemporal dementia genetics: C9ORF72, tau, progranulin and sporadics. Brain 2012, 135:794-806

10. Pereira JMS, Williams GB, Acosta-Cabronero J, Pengas G, Spillantini MG, Xuereb $\mathrm{JH}$, Hodges JR, Nestor PJ: Atrophy patterns in histologic vs clinical groupings of frontotemporal lobar degeneration. Neurology 2009, 72:1653-1660.

11. Wechsler D: Manual for the Wechsler Adult Intelligence Scale New York: Psychological Corporation; 1981.

12. Wechsler D: Wechsler Abbreviated Scale of Intelligence Psychological Corporation; San Antonio, Texas; 1999

13. Weigl $\mathrm{E}$ : On the psychology of the so called processes of abstraction. J Abnorm Soc Psychol 1927, 36:3-33.

14. Stroop J: Studies of interference in serial verbal reactions. J Exp Psychol 1935, 18:643-662.

15. Burgess PW, Shallice T: Response suppression, initiation and strategy use following frontal lobe lesions. Neuropsychologia 1996, 34:263-272.

16. Warrington E: Manual for the Recognition Memory Test for Words and Faces Windsor, UK: NFER-Nelson; 1984

17. McKenna P, Warrington EK: Testing for nominal dysphasia. J Neurol Neurosurg Psychiatry 1980, 43:781-788.

18. Oldfield $R$, Wingfield $A$ : Response latencies in naming objects. Q J Exp Psychol 1965, Series A:273-281.

19. Warrington E, James M: The Visual Object and Space Perception Battery Bury St Edmunds, UK: Thames Valley Test Company; 1991.

20. Jackson M, Warrington EK: Arithmetic skills in patients with unilateral cerebral lesions. Cortex 1986, 22:611-620.

21. Baxter D, Warrington E: Measuring dysgraphia: a graded-difficulty spelling test. Behav Neurol 1994, 3-4:107-116.

22. Freeborough PA, Fox NC, Kitney Rl: Interactive algorithms for the segmentation and quantitation of 3-D MRI brain scans. Comput Methods Programs Biomed 1997, 53:15-25.

23. Leung KK, Clarkson MJ, Bartlett JW, Clegg S, Jack CR, Weiner MW, Fox NC, Ourselin S: Robust atrophy rate measurement in Alzheimer's disease using multi-site serial MRI: tissue-specific intensity normalization and parameter selection. Neurolmage 2010, 50:516-523.

24. Statistical Parametric Mapping. [http://www.fil.ion.ucl.ac.uk/spm]

25. Ridgway G, Barnes J, Pepple T, Fox N: Estimation of total intracranial volume; a comparison of methods. Alzheimer's Dementia 2011, 7:S62-S63.

26. Reuter M, Schmansky NJ, Rosas HD, Fischl B: Within-subject template estimation for unbiased longitudinal image analysis. Neurolmage 2012, 61:1402-1418.

27. Desikan RS, Ségonne F, Fischl B, Quinn BT, Dickerson BC, Blacker D, Buckner RL, Dale AM, Maguire RP, Hyman BT, Albert MS, Killiany RJ: An automated labeling system for subdividing the human cerebral cortex on MRI scans into gyral based regions of interest. Neurolmage 2006, 31:968-980.

28. Freeborough PA, Fox NC: Modeling brain deformations in Alzheimer disease by fluid registration of serial 3D MR images. J Comput Assist Tomogr 1998, 22:838-843.

29. Knopman DS, Jack CR Jr, Kramer JH, Boeve BF, Caselli RJ, Graff-Radford NR, Mendez MF, Miller BL, Mercaldo ND: Brain and ventricular volumetric changes in frontotemporal lobar degeneration over 1 year. Neurology 2009, 72:1843-1849.

30. Gordon E, Rohrer JD, Kim LG, Omar R, Rossor MN, Fox NC, Warren JD: Measuring disease progression in frontotemporal lobar degeneration: a clinical and MRI study. Neurology 2010, 74:666-673.

31. Beck J, King A, Scahill R, Warren JD, Fox NC, Rossor MN, Collinge J, Mead S: A distinct clinical, neuropsychological and radiological phenotype is associated with progranulin gene mutations in a large UK series. Brain 2008, 131:706-720

32. Bigler ED, Maxwell WL: Neuroimaging and neuropathology of TBI. NeuroRehabilitation 2011, 28:63-74.

33. Boeve BF, Boylan KB, Graff-Radford NR, DeJesus-Hernandez M, Knopman DS, Pedraza O, Vemuri P, Jones D, Lowe V, Murray ME, Dickson DW, Josephs KA, Rush BK, Machulda MM, Fields JA, Ferman TJ, Baker M, Rutherford NJ, Adamson J, Wszolek ZK, Adeli A, Savica R, Boot B, Kuntz KM, Gavrilova R, 
Reeves A, Whitwell J, Kantarci K, Jack CR Jr, Parisi JE, et al: Characterization of frontotemporal dementia and/or amyotrophic lateral sclerosis associated with the GGGGCC repeat expansion in C9ORF72. Brain 2012, $135: 765-783$

34. Schmahmann J, Pandya D: Disconnection syndromes of basal ganglia, thalamus, and cerebrocerebellar systems. Cortex 2008, 44:1037-1066.

35. Middleton F, Strick P: Anatomical evidence for cerebellar and basal ganglia involvement in higher cognitive function. Science 1994, 266:458-461.

36. Jendroska K, Heinzel FP, Torchia M, Stowring L, Kretzschmar HA, Kon A, Stern A, Prusiner SB, DeArmond SJ: Proteinase-resistant prion protein accumulation in Syrian hamster brain correlates with regional pathology and scrapie infectivity. Neurology 1991, 41:1482-1490.

37. Velayos $J$, Oliva M, Alfageme F: Afferent projections to the mediodorsal and anterior thalamic nuclei in the cat. Anatomical-clinical correlations. Brain Pathol 1998, 8:549-552.

38. Raj A, Kuceyeski A, Weiner M: A network diffusion model of disease progression in dementia. Neuron 2012, 73:1204-1215.

39. Murray ME, Dejesus-Hernandez M, Rutherford NJ, Baker M, Duara R, GraffRadford NR, Wszolek ZK, Ferman TJ, Josephs KA, Boylan KB, Rademakers R, Dickson DW: Clinical and neuropathologic heterogeneity of C9FTD/ALS associated with hexanucleotide repeat expansion in C9ORF72. Acta Neuropathologica 2011, 122:673-690.

\section{doi:10.1186/alzrt144}

Cite this article as: Mahoney et al:: Longitudinal neuroimaging and neuropsychological profiles of frontotemporal dementia with C9ORF72 expansions. Alzheimer's Research \& Therapy 2012 4:41.

\section{Submit your next manuscript to BioMed Central and take full advantage of:}

- Convenient online submission

- Thorough peer review

- No space constraints or color figure charges

- Immediate publication on acceptance

- Inclusion in PubMed, CAS, Scopus and Google Scholar

- Research which is freely available for redistribution

Submit your manuscript at www.biomedcentral.com/submit 